\title{
Diagnóstico diferencial dos tumores hepáticos benignos: uma revisão narrativa
}

\author{
Differential diagnosis of benign liver tumors: a narrative review
}

Diagnóstico diferencial de los tumores hepáticos benignos: una revisión narrativa

Thamires Politano de Sant'Anna Alves ${ }^{1 *}$, Adriana Rodrigues Ferraz.

\begin{abstract}
RESUMO
Objetivo: Evidenciar os principais aspectos clínicos, histológicos e radiológicos implicados no diagnóstico diferencial dos principais tumores hepáticos benignos. Revisão bibliográfica: Apesar de sua natureza benigna, os tumores hepáticos benignos podem levar a complicações e desfechos graves. De forma que apesar tal entidade se apresentar assintomática na maior parte dos casos, torna-se de suma importância a realização de adequada e criteriosa conduta para chegar ao diagnóstico diferencial. De forma geral, os adenomas podem ser diferenciados das demais lesões por surgir em um contexto clínico de uso principalmente de anticoncepcional oral e se apresentar como uma lesão sólida e bem delimitada. Já a hiperplasia nodular focal caracteriza-se classicamente por apresentar cicatriz central. Os hemangiomas se apresentam por lesões bem delimitadas, sólidas, única ou múltiplas, hipervascularizadas e de localização periférica. Os Cistos se evidenciam através de uma imagem anecoica, com reforço de parede posterior e formato esférico ou oval. Considerações finais: A avaliação de lesões hepáticas pode ser desafiadora quando não vista de forma global, no entanto a literatura demonstra que quando avaliadas através da união de dados clínicos, histológicos, laboratoriais e de imagem as peculiaridades de cada lesão se tornam evidentes, permitindo o correto diagnóstico e manejo clínico.
\end{abstract}

Palavras-chave: Tumores hepáticos, Lesões benignas, Fígado.

\begin{abstract}
Objective: To highlight the main clinical, histological and radiological aspects involved in the differential diagnosis of the main benign liver tumors. Bibliographic review: Despite their benign nature, benign liver tumors can lead to complications and serious outcomes. Despite the fact that this entity is asymptomatic in most cases, it is of utmost importance to perform an appropriate and careful management to reach the differential diagnosis. In general, adenomas can be differentiated from other lesions by appearing in a clinical context of oral contraceptive use and presenting as a solid and well-defined lesion. Focal nodular hyperplasia, on the other hand, is classically characterized by a central scar. The hemangiomas are well delimited, solid, single or multiple, hypervascularized lesions located peripherally. Cysts are evidenced by an anechoic image, with posterior wall reinforcement and spherical or oval shape. Final considerations: The evaluation of liver lesions can be challenging when not seen globally, however the literature shows that when evaluated by the union of clinical, histological, laboratory and imaging findings the peculiarities of each lesion become evident, allowing the correct diagnosis and clinical management.
\end{abstract}

Keywords: Hepatic tumors, Benign lesions, Liver.

\section{RESUMEN}

Objetivo: Destacar los principales aspectos clínicos, histológicos y radiológicos implicados en el diagnóstico diferencial de los principales tumores hepáticos benignos. Revisión bibliográfica: A pesar de su naturaleza benigna, los tumores hepáticos benignos pueden dar lugar a complicaciones y resultados graves. Por lo que a pesar de que esta entidad es asintomática en la mayoría de los casos, es de suma importancia realizar un manejo adecuado y cuidadoso para llegar al diagnóstico diferencial. En general, los adenomas pueden diferenciarse de otras lesiones, ya que surgen en un contexto clínico de uso de anticonceptivos orales

\footnotetext{
1 Universidade de Vassouras (UV), Vassouras - RJ. *E-mail: poliatano.thamires@gmail.com
} 
principalmente y se presentan como una lesión sólida y bien definida. Por otro lado, la hiperplasia nodular focal se caracteriza clásicamente por presentar una cicatriz central. Los hemangiomas se presentan como lesiones bien definidas, sólidas, únicas o múltiples, hipervascularizadas y de localización periférica. Los quistes se evidencian con una imagen anecoica, con refuerzo de la pared posterior y forma esférica u ovalada. Consideraciones finales: La evaluación de las lesiones hepáticas puede ser un reto cuando no se ven de forma global, sin embargo la literatura muestra que cuando se evalúa mediante la unión de datos clínicos, histológicos, de laboratorio y de imagen las peculiaridades de cada lesión se hacen evidentes, permitiendo el diagnóstico correcto y el manejo clínico.

Palabras clave: Tumores hepáticos, Lesiones benignas, Hígado.

\section{INTRODUÇÃO}

Os Tumores Hepáticos Benignos (THB) consistem em um grupo heterogêneo de lesões que podem se originar de um fígado saudável ou em associação com outras patologias hepáticas. O crescente aumento da detecção destas lesões tem ocorrido devido à ampla e indiscriminada utilização de métodos de imagens na prática clínica moderna, sendo parte das alterações detectadas incidentalmente em exames que primariamente visavam diagnosticar alterações em outros órgãos e estruturas (RONCALLI M, et al., 2016; AMARAWEERA C, et al., 2019).

Apesar do aumento no número de tumores hepáticos encontrados em exames de imagem como a Tomografia Computadorizada (TC) e na Ressonância Magnética (RM), ainda há uma significante dificuldade em diferenciar tais lesões, necessitando de uma abordagem cautelosa com análise conjunta dos aspectos clínicos, radiológicos, histopatológicos e genéticos para melhor definição do tumor (AMARAWEERA C, et al., 2019; PANQUEVA RDPL, 2015).

Quando corretamente indicados e avaliados de forma sistemática e concisa os exames de imagem se tornam uma importante ferramenta diagnóstica dos THB, permitindo um melhor direcionamento para a conduta clínica e evitando procedimentos invasivos, muitas vezes desnecessários, como a biópsia. (HUSAINY MA, et al., 2017)

As lesões hepáticas podem ser classificadas em benignas ou malignas, podendo o primeiro grupo ser dividido ainda quanto à sua origem em hepatocelular, epitélio biliar, vascular e outros. Entre os de origem hepatocelular destacam-se o Adenoma Hepatocelular (AHC), que apesar de raro possui importante relevância clínica devido seu potencial de gerar complicações e evoluir para malignidade e a Hiperplasia Nodular Focal (HNF) que é considerada a segunda lesão benigna mais comum do fígado, perdendo apenas para hemangioma (PANQUEVA RDPL, 2015; RENZULLI M, et al., 2019).

Quanto aos de origem do epitélio biliar destaca-se os cistos, que podem ser simples ou complicados, estes últimos mais raros (LANTINGA MA, et al., 2013). No grupo de origem vascular destaca-se o Hemangioma $(\mathrm{HH})$, tumor hepático benigno mais comum (RENZULLI M, et al., 2019; SOTOMAYOR JR, et al., 2016; MORTELE KJ e ROS PR, 2002).

O presente artigo se torna relevante por realizar uma revisão crítica da literatura a fim de elucidar as principais características clínicas, histopatológicas e radiológicas que permitem o diagnóstico diferencial das lesões hepáticas benignas, cada vez mais encontradas incidentalmente em exames de imagem. Teve como objetivo evidenciar os principais aspectos envolvidos para diferenciação dos principais tumores hepáticos benignos.

\section{REVISÃO BIBLIOGRÁFICA}

O diagnóstico diferencial das lesões hepáticas benignas é um desafio para clínicos e radiologistas, de forma que por abranger uma variedade ampla de patologias que surgem em sua maioria em um contexto assintomático, torna-se imperioso que tais especialistas sejam capazes de reunir dados na anamnese e nos exames laboratoriais e de imagem capazes de direcionar para o diagnóstico correto da lesão. Para cada 
possível lesão benigna encontrada há características típicas descritas na literatura analisada que permitem o direcionamento adequado para definir o diagnóstico (HUSAYNI MA, et al., 2017; PATACSIL SJ, et al., 2020; MORTELE KJ, ROS PR, 2002).

\section{Adenoma hepatocelular}

É o terceiro tipo mais comum dentre os tumores hepáticos benignos. Caracteriza-se por ser um tumor sólido, bem delimitado e com pouca ou nenhuma cápsula fibrosa. É mais comum em mulheres jovens em uso de anticoncepcional oral, sendo a incidência do AHC 30 vezes maior em usuárias da medicação em questão comparada a não usuárias. Dentre outros fatores de risco destacam-se doenças de acúmulo do glicogênio, síndrome metabólica, uso de esteroides anabolizantes e diabetes mellitus familiar (RENZULLI M, et al., 2019; SZOR DJ, et al., 2013; CUERVO C, et al., 2014).

Os AHCs eram divididos em quatro grupo de acordo com sua classificação molecular: Adenoma Hepatocelular Inflamatório (I-HCA), Adenoma Hepatocelular Associado à Mutação no Gene HNFa1 (H-HCA), Adenoma Hepatocelular Associado à Mutação $\beta$-catenina ( $\beta-\mathrm{HCA})$, sendo este ainda subdividido em éxon 3 $\beta$-catenina Mutado ( $3 \beta$-HCA) e Éxon 7-8 $\beta$-catenina mutado (7,8 $\beta$-HCA), e os adenomas hepatocelulares não classificáveis. No entanto em 2017 um estudo francês propôs uma nova classificação acrescentando duas formas mistas de I-HCA com o éxon $3 \beta$-HCA e o éxon $7,8 \beta$-HCA e o novo grupo Adenoma Hepatocelular Associado à Via Sonic Hedgehog (shHCA) familiar (RENZULLI M, et al., 2019; SZOR DJ, et al., 2013; CUERVO C, et al., 2014).

Histologicamente se caracterizam principalmente por nódulos benignos proliferativos nos hepatócitos que podem apresentar esteatose e armazenamento de glicogênio. Quanto à história clínica são comumente encontrados incidentalmente em exames de imagem como TC e RM. Quando presentes os sintomas incluem discreta dor abdominal em região epigástrica e hipocôndrio direito, distensão abdominal, massa palpável e sangramento intra-abdominal ou intratumoral. Laboratorialmente pode haver elevações das enzimas canaliculares fosfatase alcalina e gamaglutamil transferase. Em caso de degeneração maligna pode ocorrer aumento dos níveis séricos de Alfafetoproteína (PANQUEVA RDPL, 2015; SZOR DJ, et al., 2013; PATACSIL SJ, et al., 2020).

Em relação aos exames de imagem a Ultrassonografia (USG) de abdome possui dificuldade em promover o diagnóstico diferencial com outras lesões hepáticas, no entanto é possível detectar uma lesão bem delimitada, heterogênea, presença de calcificações ou área de necrose. Na TC de abdome é possível identificar lesão bem delimitada, de aspecto heterogêneo (hemorragia, necrose e fibrose), com presença de hipervascularização irregular após uso de contraste. O grande exame de imagem é a RM com utilização de agentes de contraste hepatobiliar específicos, que se mostrou superior a todos os outros na detecção dos AHCs, sendo capaz ainda de diferenciar os subtipos de adenomas em mais de $80 \%$ dos casos. Quando não há a possibilidade de chegar ao diagnóstico através de exames de imagem é feita a biópsia transpariental, porém esta é uma conduta de exceção, devido ao risco de hemorragia, bem como pela heterogeneidade intratumoral dos AHCs levando à necessidade de mais de uma biópsia para adquirir o material suficiente para análise (PANQUEVA RDPL, 2015; SZOR DJ, et al., 2013).

A complicação mais comum consiste no sangramento que pode ocorrer de forma intra-tumoral sendo mais comum no I-HCA em lesões caracteristicamente maiores de $5 \mathrm{~cm}$ e de localização subcapsular. $O$ sangramento pode se dar também por ruptura do adenoma para a cavidade levando ao quadro de hemoperitônio. Outra complicação consiste na degeneração maligna para carcinoma hepatocelular, mais comum no grupo $\beta$-HCA (SZOR DJ, et al., 2013; CUERVO C, et al., 2014).

Para quadros assintomáticos a conduta incialmente é a suspensão do uso de anticoncepcionais orais ou esteroides anabolizantes. $\mathrm{Na}$ ausência de fatores de risco para complicações a conduta é o acompanhamento regular da lesão. Pacientes sintomáticos que estejam estáveis podem ser candidatos ao tratamento conservador de acordo com a análise das características da lesão. Caso apresente ruptura levando ao quadro de instabilidade ou lesão sintomática maior que $5 \mathrm{~cm}$, deve ser instituído de forma imediata a ressecção cirúrgica ou embolização (CUERVO C, et al., 2014). 
As principais patologias que fazem diagnóstico diferencial com o AHC são a HNF e o hepatocarcinoma. Com relação à HNF o adenoma pode ser diferenciado pela sua história clínica já que geralmente surge em um contexto de uso de anticoncepcional oral, síndrome metabólica, doença do glicogênio ou uso de esteroides anabolizantes bem como pode ser diferenciado por meio de exames de imagem, de forma que o AHC difere da HNF por não apresentar cicatriz central, padrão estrelado, entre outras características típicas da HNF. Com relação ao hepatocarcinoma, toda lesão suspeita de malignidade deve ser ressecada de forma que a diferenciação só será realizada através do estudo histopatológico da peça cirúrgica (RONCALLI M, et al., 2016; PANQUEVA RDPL, 2015; RENZULLI M, et al., 2019; CUERVO C, et al., 2014).

\section{Hiperplasia nodular focal}

Consiste no segundo tumor hepático benigno mais comum e acredita-se possuir origem de uma reação hiperplásica do parênquima hepático em resposta a lesões e malformações vasculares, embora sua patogênese ainda seja controversa. Sua prevalência é maior em mulheres em idade reprodutiva, com uma proporção de 5-10 casos no sexo feminino para cada 1 no sexo masculino. A HNF se apresenta em duas formas, a clássica é caracterizada por arquitetura nodular anormal, vasos malformados e proliferação colangiolar, já a não clássica é subdividida em HNF telangiectática, HNF com atipia citológica e por fim o subtipo hiperplásico misto e HNF adenomatosa (RONCALLI M, et al., 2016; PANQUEVA RDPL, 2015; ZHANG $\mathrm{H}$, et al., 2016; MORTELE KJ, ROS PR, 2002).

Histologicamente caracteriza-se por nódulos benignos organizados em trabéculas com duas a três células, separadas por septos fibrosos contendo infiltrado inflamatório e presença de metaplasia ductular que se reúnem em uma cicatriz central composta por estruturas vasculares de paredes espessas e de formas e tamanhos variados. Em torno de $25 \%$ dos casos, pode haver presença de esteatose associada a alterações xantomatosas de hepatócitos periseptares. Macroscopicamente configura uma lesão sólida, única em aproximadamente $80 \%$ dos casos, geralmente menor que $5 \mathrm{~cm}$ pálida, bem delimitada, com ausência de capsula fibrosa, em geral apresentando cicatriz central (RONCALLI M, et al., 2016; PANQUEVA RDPL, 2015; ZHANG H, et al., 2016).

A HNF na maioria das vezes surge em um contexto clínico assintomático, sendo descoberta incidentalmente através de exames de rotina. Quando a sintomatologia está presente se manifesta por dor abdominal, perda de peso, mal-estar ou massa palpável. O crescimento da lesão é raro e embora tenha sido apontada uma associação com uso de anticoncepcionais orais, estudos demonstram que a HNF não é um tumor hormônio dependente, porém o uso da medicação pode levar ao crescimento tumoral (RONCALLI M, et al., 2016; PANQUEVA RDPL, 2015; ZHANG H, et al., 2016; HAU HM, et al., 2015).

Quanto aos exames de imagem, apresenta à UGS como uma massa ecogênica e em $20 \%$ dos casos de padrão estrelado. Na TC é possível identificar lesão hipodensa com realce homogêneo na fase arterial. $O$ método de imagem de escolha é a RM com contraste devido sua maior sensibilidade comparada aos demais, por meio de tal exame é possível distinguir a HNF de outras lesões focais. O diagnóstico por meio de biópsia é indicado somente quando não for possível obtê-lo através da história clínica associada a exames de imagem, e ainda assim é conclusivo em apenas $25 \%$ a $50 \%$ dos casos (RONCALLI M, et al., 2016; PANQUEVA RDPL, 2015; ZHANG H, et al., 2016; HAU HM, et al., 2015).

O tratamento inicial de escolha para pacientes assintomáticos é o conservador, com acompanhamento regular. Nos casos em que não é possível descartar malignidade, na presença de sintomas, manifestações atípicas ou naqueles em que há qualquer alteração no número, tamanho ou sintomas das lesões durante o acompanhamento clínico, o tratamento consiste na ressecção cirúrgica (RONCALLI M, et al., 2016; HAU HM, et al., 2015).

A HNF faz diagnóstico diferencial com AHC e com hiperplasia nodular regenerativa (HNR). A diferenciação entre HNF e AHC se dá através da história clínica bem como do estudo de imagem, no qual a HNF possui características típicas como presença de cicatriz central e padrão estrelado. Já a diferenciação entre HNF e HNR é possível através da história clínica, pois a HNR surge em um contexto de doença sistêmica como 
artrite reumatoide, doença celíaca, lúpus eritematoso sistêmico, policitemia vera, entre outras, além de se apresentar com quadro de hipertensão porta não cirrótica e possuir macroscopia semelhante à cirrose o que não é característico da HNF (RONCALLI M, et al.,2016; PANQUEVA RDPL, 2015).

\section{Hemangioma}

É considerado o tumor hepático benigno mais comum e apesar de sua fisiopatologia ainda controversa é sabidamente originado de uma malformação vascular. Possui incidência entre $0,4 \%$ e $20 \%$ na população geral, de maneira que o sexo feminino é o mais acometido, em uma proporção de 5:1 em relação ao masculino. Apesar de sua origem vascular, pode apresentar seu crescimento associado à exposição contínua de estrogênio e progesterona. São classificados em cavernosos, capilares ou esclerosantes, de forma que 0 primeiro grupo é o mais comum (PATACSIL SJ, et al., 2020; LEON M, et al., 2020; FODOR M, et al., 2018).

Histologicamente possui origem do mesoderma, sendo revestido por uma única camada de células endoteliais, apresenta septos fibrosos e é alimentado pela circulação arterial hepática. Macroscopicamente se apresentam por lesões bem delimitadas, sólidas, única ou múltiplas, hiper vascularizadas e de localização periférica. Em geral são pequenas, menores que $5 \mathrm{~cm}$, no entanto podem se apresentar como hemangioma gigante, quando se apresenta com $5 \mathrm{~cm}$ ou mais, e atualmente estudos têm sugerido o termo hiper ou mega gigantes para hemangiomas maiores que $10 \mathrm{~cm}$ (PANQUEVA RDPL, 2015; LEON M, et al., 2020; FODOR M, et al., 2018).

De forma geral o quadro é assintomático, descoberto incidentalmente através de exames de imagem. Os sintomas são mais prevalentes em lesões maiores que $5 \mathrm{~cm}$ e são inespecíficos, representados por quadro de dor abdominal e desconforto em quadrante superior direito, plenitude pós-prandial, náuseas e vômitos, secundários à distensão e inflamação da cápsula de Glisson. Menos comumente pode se associar a febre, icterícia, dispneia, insuficiência cardíaca de alto débito e hemobilia. Pode haver também, a depender da localização da lesão, presença de compressão de estruturas adjacentes causando outros sintomas a depender da estrutura acometida. Os HHs gigantes podem ainda complicar com a Síndrome de KasabachMerrit. Mais raramente pode ocorrer ruptura da lesão, seja de maneira traumática ou espontânea, levando ao quadro de hemorragia (LEON M, et al.,2020; ZHAI L, et al., 2019).

O diagnóstico é feito através de exames de imagem, em geral a lesão se apresenta por meio de realce nodular periférico e preenchimento centrípeto progressivo. O USG é o exame inicial de escolha e demonstra lesão hiperecoica com realce acústico posterior. O Ultrassom com Contraste Aprimorado (CEUS) possui maior especificidade. Atualmente o melhor método de imagem para diagnóstico de $\mathrm{HH}$ é a RM, sendo possível identificar uma lesão bem delimitada, hipointensa em T1 e hiperintensa em T2. Quadros atípicos de HH a angiografia evidencia imagem em "árvore com neve" ou "algodão" em conjunto com um grande vaso de alimentação e agrupamento difuso de contraste. Quanto à biópsia da lesão, esta não é indicada devido baixa acurácia diagnóstica e risco de hemorragia (PATACSIL SJ, et al.,2020; LEON M, et al., 2020).

OS HHs pequenos e assintomáticos não necessitam de tratamento e acompanhamento. Já a conduta para lesões maiores que $5 \mathrm{~cm}$ consiste em acompanhar o seu padrão de crescimento, em caso de alterações significativas o tratamento deve ser instituído. Como modalidade de tratamento não cirúrgico têm-se a embolização arterial transcateter (EAT), a ablação por radiofrequência e a ablação por microondas que podem ser utilizadas como tentativa de resolução do quadro antes de seguir para a cirurgia. Quanto às modalidades de tratamento cirúrgico temos: Hepatectomia, que possui grande indicação em HH de localização central; Enucleação que se encontra bem indicada para $\mathrm{HH}$ de localização periférica; Ligadura da artéria hepática e por fim o transplante hepático que possui indicação nos casos de $\mathrm{HH}$ gigantes irressecáveis, falha terapêuticas com as modalidades já citadas ou em complicações que levem a risco de vida (LEON M, et al., 2020; KETCHUM WA, et al., 2019).

Os HHs possuem como diagnóstico diferencial peliose hepática, angiossarcoma, hamartoma esclerótico do ducto biliar, bem como outras lesões benignas do fígado que quando sintomáticas se apresentam com sintomatologia semelhante, no entanto através da criteriosa análise dos exames de imagem é possível determinar com precisão seu diagnóstico (PANQUEVA RDPL, 2015; LEON M, et al., 2020). 


\section{Cistos hepáticos}

Apesar de não se caracterizar como um tumor propriamente dito, os cistos simples hepáticos (CSH) são a lesão mais comum do fígado, sendo encontrados em 2,5 a 18\% da população geral, o que torna de grande importância sua diferenciação das lesões tumorais supracitadas. Acredita-se que os CSH se originam de malformações congênitas da placa ductal no período embrionário, surgindo através de ductos biliares aberrantes, de forma que sejam revestidos por epitélio do ducto biliar e possuam conteúdo líquido claro similar à bile (FURUMAYA A, et al., 2020; BERNTS LHP, et al., 2019; ZHANG H, et al., 2016).

Em geral se apresenta como lesão pequena e assintomática, de crescimento lento, encontrada incidentalmente em exames de imagem. Quando presentes os sintomas incluem dor ou desconforto abdominal, náuseas, vômitos, perda de apetite, saciedade precoce e dispneia, que de maneira geral ocorrem em grandes cistos, maiores que $5 \mathrm{~cm}$, bem como massa palpável e hepatomegalia ao exame físico. Os $\mathrm{CHS}$ podem apresentar como complicação hemorragia intracística, infecção, ruptura, hipertensão portal, obstrução biliar e icterícia. Laboratorialmente pode haver elevação da gama glutamil transferase (GGT), de antígeno carcinoembrionário (CEA) e de antígeno CA 19-9 (BERNTS LHP, et al., 2019; ZHANG H, et al., 2016; 19; RAN B, et al., 2019).

Quanto aos exames de imagem à USG é possível identificar imagem anecoica, com ausência de septações, reforço de parede posterior, com cisto de forma esférica ou oval. A TC se apresenta com lesão hipodensa e homogênea. Já a RM evidencia hipointensidade em T1 e hiperintensidade em T2, que não se altera após o uso de contraste. A USG tem maior precisão diagnóstica, sendo o exame inicial de escolha. Em caso de complicações o grande exame é o CEUS, pois a hemorragia intracística ao USG poderia simular presença de septos ou porções sólidas, porém através do CEUS é possível visualizar o fluxo vascular dentro de septos e componentes sólidos, ausentes no cisto simples com hemorragia intracística (BERNTS LHP, et al., 2019; ZHANG H, et al; 2016; RAN B, et al., 2019; NEIJENHUIS MK, et al., 2019).

O tratamento de cistos pequenos e assintomáticos é feito de forma conservadora, através de acompanhamento clínico. Cistos grandes e sintomáticos podem ser tradados por meio de fenestração laparoscópica, escleroterapia por aspiração percutânea ou omentopexia (BERNTS LHP, et al., 2019; ZHANG H, et al., 2016; RAN B, et al., 2019; NEIJENHUIS MK, et al., 2019).

Os CHS fazem diagnóstico diferencial com Equinococose, uma importante zoonose que pode se manifestar através de uma forma cística. A diferenciação se dá através da história clínica de região endêmica na Equinococose, além dos exames de imagem como USG e TC característicos da zoonose e teste sorológico positivo. Outros importantes diagnósticos diferenciais incluem cistadenoma e cistadenocarcinoma que podem facilmente ser diferenciadas dos CHS através do USG, que diferente do que ocorre nos CHS, evidencia lesão de borda irregular, hipoecogênica, com presença de septações e componentes sólidos hiperecogênicos (ZHANG H, et al., 2016; RAN B, et al., 2019).

Há ainda uma entidade que ocorre quando há a presença de mais de 10-20 cistos hepáticos, denominada Doença Policística Hepática (DPH), que se encontra associada às desordens genéticas conhecidas como Doença Hepática Policística Autossômica Dominante (DHPAD) e Doença Renal Policística Autonômica Dominante (DRPAD) (BERNTS LHP, et al., 2019; ZHANG H, et al., 2016; ABU-WASEL B, et al., 2013).

\section{CONSIDERAÇÕS FINAIS}

Com o avanço das inovações tecnológicas na área da saúde, novas modalidades de exames radiológicos vêm surgindo ou sendo aprimoradas, tornando crescente a requisição destes na prática clínica. A avaliação de lesões hepáticas, encontradas incidentalmente em exames de imagem, pode ser desafiadora quando não analisada de forma global, buscando além das características visualizadas e laudadas, a pesquisa de dados clínicos, histológicos e laboratoriais que juntos corroborem para o diagnóstico correto da lesão. Dessa forma, a literatura apresentada demonstra que os principais tumores hepáticos benignos se comportam de maneira única e peculiar dentro de cada grupo e assim conclui-se que com uma criteriosa investigação e análise conjunta dos dados encontrados é possível determinar o tipo de lesão e seu devido manejo clínico. 


\section{REFERÊNCIAS}

1. ABU-WASEL B, et al. Pathophysiology, epidemiology, classification and treatment options for polycystic liver diseases. World J Gastroenterol, 2013; 19(35): 5775-5786.

2. AMARAWEERA C, et al. Systematic approach for benign liver lesions in non - cirrhotic livers. Ceylon Med J, 2019; 64(2): 66-69.

3. BERNTS LHP, et al. Clinical response after laparoscopic fenestration of symptomatic hepatic cysts: a systematic review and meta-analysis. Surgical Endoscopy, 2019; 33: 691-704.

4. CUERVO C, et al. Hepatocellular Adenomas: Current Findings in Images Which Allow its Characterization and Management. Revista Colombiana de Radiologia, 2014; 25(2): 3934-3941.

5. FODOR M, et al. Indications for liver surgery in benign tumours. European Surgery, 2018; 50: 125-131.

6. FURUMAYA A, et al. Systematic review on percutaneous aspiration and sclerotherapy versus surgery in symptomatic simple hepatic cysts. HPB (Oxford), 2020; 23(1): 11-24.

7. HAU HM, et al. The value of liver resection for focal nodular hyperplasia: resection yes or no?. European Journal of Medical Research, 2015; 20: 86.

8. HUSAYNI MA, et al. Typical and atypical benign liver lesions: A review. Clin Imaging, 2017; 44: 79-91.

9. KETCHUM WA, et al. Management of Symptomatic Hepatic "Mega" Hemangioma. Hawaii J Med Public Health, 2019; 78(4): 128-131.

10. LANTINGA MA, et al. Evaluation of hepatic cystic lesions. World J Gastroenterol, 2013; 19(23): 3543-3554.

11. LEON M, et al. Hepatic hemangioma: What internists need to know. World J Gastroenterol, 2020; 26(1): 11-20.

12. MORTELE KJ, ROS PR. Benign liver neoplasms. Clinical Liver Disease, 2002; 6(1): 119-145.

13. NEIJENHUIS MK, et al. Symptom relief and not cyst reduction determines treatment success in aspiration sclerotherapy of hepatic cysts. European Radiology, 2019; 29: 3062-3968.

14. PANQUEVA RDPL. Diagnosis and Differential Diagnosis Problems with Histological Variants of Benign Liver Neoplasms. Revista Colombiana de Gastroenterologia, 2015; 30(1): 116-124

15. PATACSIL SJ, et al. A Review of Benign Hepatic Tumors and Their Imaging Characteristics. Cureus, 2020; 20(1): e6813.

16. RAN B, et al. Differentiation between hepatic cystic echinococcosis types 1 and simple hepatic cysts. Medicine, 2019; 98: 1.

17. RENZULLI M, et al. Hepatocellular adenoma: An unsolved diagnostic enigma. World J Gastroenterol, 2019; 25(20): 2442-2449.

18. RONCALLI M, et al. Benign hepatocellular nodules of healthy liver: focal nodular hyperplasia and hepatocellular adenoma. Clinical and Molecular Hepatology, 2016; 22(2): 199-211.

19. SOTOMAYOR JR, et al. Giant hepatic hemangioma. Cirugía paraguaya, 2016; 40(2): 34-35

20. SZOR DJ, et al. Adenoma Hepático. Arquivos Brasileiros de Cirurgia Digestiva, 2013; 26(3): $219-222$.

21. ZHAI L, et al. Spontaneous rupture of giant hepatic hemangioma: misdiagnosis as gastrointestinal perforation. Journal of International Medical Research, 2019; 47(9): 4514-4521.

22. ZHANG H, et al. Evaluation of the characteristics of hepatic focal nodular hyperplasia: correlation between dynamic contrast-enhanced multislice computed tomography and pathological findings. Onco Targets and Therapy, 2016; 9: 5217-5224. 